Socioeconomic status and risk of incident atrial fibrillation; Page 1

\title{
Association between Socio-Economic Status and Incident Atrial Fibrillation
}

Satish Ramkumar MBBS BMedSci MMed ${ }^{1,2,3}$, Ayame Ochi BMedSci ${ }^{4}$, Hong Yang BMed ${ }^{4}$, Nitesh Nerlekar MBBS, MPH'1,3, Nicholas D’Elia MBBS BMedSci(Hons) ${ }^{1}$, Elizabeth L Potter MBBS BSc ${ }^{1,2}$, Isabella C Murray $^{4}$, Nishee Nattraj ${ }^{4}$, Ying Wang BMed ${ }^{4}$, Thomas H Marwick MBBS, PhD, MPH ${ }^{1,2,4}$

\author{
${ }^{1}$ Baker Heart and Diabetes Institute, 75 Commercial Road Melbourne, Victoria 3004, Australia; ${ }^{2}$ School of \\ Public Health and Preventative Medicine, Monash University, 99 Commercial Road, Melbourne, Victoria 3004, \\ Australia; ${ }^{3}$ Monash Heart, Monash Cardiovascular Research Centre, 246 Clayton Road, Clayton, Victoria 3168, \\ Australia; ${ }^{4}$ Menzies Institute for Medical Research, 17 Liverpool Street Hobart, Tasmania 7000, Australia;
}

Declarations of Interest and contributions:

S.R - Involved in study design, patient recruitment, data collection, analysis and manuscript preparation. Receives equipment and software support from Semacare Inc, a manufacturer of handheld ECG devices. The sponsors had no role in the design and conduct of the study, in the collection, analysis, and interpretation of the data, and in the preparation, review, or approval of the manuscript. Receives research scholarships from the Heart Foundation and Avant

T.H.M - Involved in study design, data analysis, manuscript preparation and overall project supervision. Receives equipment and software support from Semacare Inc, a manufacturer of handheld ECG devices. The sponsors had no role in the design and conduct of the study, in the collection, analysis, and interpretation of the data, and in the preparation, review, or approval of the manuscript.

AO - Involved in data collection, patient reviews, data analysis and manuscript preparation.

HY - receives research scholarship from the Heart Foundation. Performed echocardiograms. Involved in data collection, patient reviews, data analysis and manuscript editing.

$\mathrm{NN}$ - Involved in data and statistical analysis as well as manuscript preparation and editing.

ND - Involved in data collection, patient reviews, data analysis and manuscript preparation.

ELP - Involved in data collection, patient reviews, data analysis and manuscript preparation. IM - Involved in data collection, ECG analysis, data analysis and manuscript editing.

NN- Involved in data collection, ECG analysis, data analysis and manuscript editing.

YW - Involved in data collection, patient reviews, data analysis and manuscript preparation.

Running title: Socio-economic status and risk of incident atrial fibrillation

Corresponding Author: $\quad$ Prof. Thomas H. Marwick

Baker Heart and Diabetes Institute,

75 Commercial Road, Melbourne, 3004, Australia

Phone: +61-3-8532-1550; Fax: +61-3-8532-1160

Email: Tom.Marwick@baker.edu.au

This is the author manuscript accepted for publication and has undergone full peer review but has not been through the copyediting, typesetting, pagination and proofreading process, which may lead to differences between this version and the Version of Record. Please cite this article as doi: 10.1111/imj.14214 
Key words; socioeconomic status, atrial fibrillation, screening

Abstract 246 words; Text 3000 words; References 29; Figures 1; Tables 3

\begin{abstract}
Background: Low socio-economic status is associated with cardiovascular diseases, and an association with atrial fibrillation(AF) could guide screening. We investigated if indices of disadvantage(IAD), education/occupation(IEO) and economic resources(IER) were associated with incident AF, independent of risk factors and cardiac function.
\end{abstract}

Methods: We studied community-based participants aged $\geq 65$ years with AF risk factors(n=379, age $70 \pm 4$ years, $45 \%$ men). The CHARGE-AF score (a well validated AF risk score) was used to assess 5year risk of developing AF. Participants also had baseline echocardiograms. IAD, IEO and IER were obtained from the 2011 Socio-Economic Indexes for Areas(SEIFA) score, in which higher decile ranks indicate more advantaged areas. Patients were followed up for incident AF(median 21 (range 531) months), with AF diagnosed by clinical review including 12 lead ECG, as well as single lead portable ECG monitoring used to record 60 second ECG tracings five times/day for one week. Cox proportional hazards models were used to assess the association between socio-economic status and incident AF.

Results: Subjects with $\mathrm{AF}(\mathrm{n}=50,13 \%)$ were more likely to be male(64\% vs.42\%, $\mathrm{p}=0.003)$ and had higher CHARGE-AF score(median 7.1\%(5.2-12.8\%) vs. 5.3\%(3.3-8.6\%), $\mathrm{p}<0.001)$. Areas with lower socio-economic status(IAD and IEO) had a higher risk of incident AF independent of LV function and CHARGE-AF score(HR for IAD 1.16, 95\% C.I 1.05-1.29, p=0.005 and HR for IEO 1.18, 95\% C.I 1.07-1.30, $\mathrm{p}=0.001)$. 
Conclusion: Regional socio-economic status is associated with risk of incident AF, independent of LV function and clinical risk. This association might permit better regional targeting of prevention.

Keywords: Atrial fibrillation, screening, socioeconomic status

Atrial fibrillation (AF) is associated with stroke, heart failure, increased all-cause mortality, ${ }^{1,2}$ and substantial financial cost. ${ }^{3,4}$ The epidemics of obesity, diabetes mellitus and metabolic syndrome have been associated with the increasing prevalence of $\mathrm{AF}$, which has become a significant population health problem. ${ }^{5-7}$ The early diagnosis of AF may lead to individualized lifestyle intervention ${ }^{8}$ and anticoagulation, and these steps may be associated with a reduction in complications and health care costs. The development of hand-held electrocardiogram (ECG) screening devices ${ }^{9-15}$, has increased the feasibility of AF screening. Appropriate selection of patients for screening is of critical importance; the development of a risk assessment score, ${ }^{16,17}$ based upon the link between AF and clinical risk factors, is an important component of identifying individual patients at risk. The introduction of a screening program should also involve consideration of which communities are at risk.

Socio-economic deprivation is strongly associated with increased risk of metabolic syndrome and coronary artery disease, ${ }^{18-20}$ beyond its association with reduced health care access. Low household income influences dietary choices, psychological well-being and is associated with a sedentary lifestyle. ${ }^{21}$ Poor literacy and education levels may affect treatment adherence and risk awareness. 
There may also be higher rates of substance and alcohol abuse in deprived areas. ${ }^{22}$ However, the association between socioeconomic deprivation and AF risk is controversial, with reports of an association of lower household income with increased $\mathrm{AF}$ risk, ${ }^{23}$ balanced by other evidence that neighborhood deprivation and socioeconomic disparities were not independently associated with AF. ${ }^{24}$ The purpose of this study was to assess the association of regions of socioeconomic deprivation with risk of incident AF.

\section{Methods.}

Study population. This prospective observational cohort study recruited participants from both urban and rural settings in Tasmania (an island located south of the Australian mainland) and Victoria (a larger State in the south of mainland Australia). Apart from the major cities (Hobart and Launceston), much of Tasmania is geographically isolated, with limited access to health care. ${ }^{25}$ Participants from the community $\geq 65$ years were recruited if they had 1 or more AF/heart failure (HF) risk factors, including hypertension (systolic BP $>140 \mathrm{mmHg}$ or pre-existing use of anti-hypertensive medications), type 2 diabetes mellitus (T2DM, based on self-report of diagnosis or the current use of diabetic medications), and obesity (body mass index $\geq 30$ ). Subjects were excluded if they were unable to provide written consent, had known HF or left ventricular (LV) systolic dysfunction, moderate/severe valvular disease or life expectancy $<1$ year. All patients with a history of AF and anticoagulated or were noted to have AF during baseline 12 lead ECG and echocardiograms were excluded. All patients were provided written informed consent and the study protocol conforms with the ethical guidelines of the 1975 Declaration of Helsinki and approval was obtained from the Tasmanian Human Research Ethics Committee (HREC project number H0013333). The study was registered on the Australian and New Zealand clinical trials registry (ACTRN12614000080628). 
Clinical findings. Participants provided a clinical history and answered questionnaires to assess overall health status at the start of the study. Information regarding demographics, past medical history, medication history as well as baseline examination data (height, weight, body mass index [BMI], blood pressure [BP]) was recorded for all participants. Baseline 12 lead ECG and echocardiography were conducted in all participants, and patients with previously unrecognized HF were excluded. Assessment of AF risk was performed using the CHARGE-AF score. ${ }^{17}$ Exercise capacity was assessed using the six-minute walk test.

Assessment of socioeconomic status. All participants had information collected on education level. The Socio-Economic Indexes for Areas (SEIFA) score is derived from several domains of national census data (including education, housing, household income, employment and occupation) to provide a multidimensional assessment of socioeconomic status based on postcode. This was used to describe the regional variations of participants' overall socioeconomic status. ${ }^{25}$

The SEIFA score has three main indices; an index of advantage/disadvantage (IAD, based on income, occupation and housing), an index of education/occupation (IEO, based on education and occupation) and an index of economic resources (IER, based on individual income, mortgage repayments, rental return and family income). These indices are expressed to deciles, with the lowest scoring $10 \%$ of areas given a decile number of 1 and the highest $10 \%$ of areas are given a decile number of 10 . Hence, higher scores reflect more advantaged areas..$^{25}$

AF follow-up. AF was diagnosed using multiple detection methods. All participants had baseline and follow-up assessment including a 12-lead ECG and echocardiogram. In the interim, any patients diagnosed with AF by local physicians were documented. Screening for subclinical AF was performed using a single lead ECG device (Remon RM-100, Semacare, China). The single lead device was used to record 60 second single lead ECG tracings using three points of finger contact with electrodes, five times per day for a one week (i.e. 35 recordings). ECG recordings were then 
exported as PDF files for interpretation, and all were assessed by a physician. The presence of AF (an irregular rhythm of $\geq 30 \mathrm{sec}$ with a variable R-R interval and absent $\mathrm{P}$ waves) was confirmed by two independent physicians who were blinded to the patient's clinical details. The patient was advised of the recognition of subclinical AF, and further management and investigation was provided by their usual medical practitioner.

Outcome measures: Our primary outcome measure was the overall proportion of the cohort with new onset AF during the follow-up period.

Statistical analysis. All categorical variables are presented as frequencies/percentages and continuous variables presented as means/standard deviation (if normally distributed) or medians/inter-quartile range (if non-parametric). Patients with incident AF were compared to those remaining in sinus rhythm. Groups were compared using the chi square test for categorical data and the independent two sample t-test for continuous data. Patients were grouped according to the SEIFA rank ( $<5$ vs. $\geq 5)$ and Nelson-Aalen cumulative hazard estimate plots were constructed, and the log-rank test used to assess the differences between curves. A Cox proportional hazards regression analysis was used to calculate the adjusted hazard ratios (HR) and 95\% confidence intervals for the association between each socioeconomic index and incident AF. Clinically relevant model covariates included CHARGE-AF score, global longitudinal strain, gender and indexed left atrial volume. The follow up time was the time from the initial baseline clinical assessment to the completion of portable device screening or the date of diagnosis of AF (whichever came first). Analyses were considered to be statistically significant if two-tailed $\mathrm{p}$ values were $<0.05$. Statistical analysis was performed using SPSS v.22 (SPSS, Chicago, IL) and Stata v.13 (StataCorp, College Station, TX).

\section{Results}


Patient characteristics. The baseline characteristics of the 379 subjects included in the study (mean age $70 \pm 4$ years, $45 \%$ male) are summarized in Table 1 . Thirteen participants (3\%) had a previous diagnosis of paroxysmal AF. Cardiovascular risk factors (including T2DM, obesity, hypercholesterolemia and hypertension) were highly prevalent. There was a large proportion of participants with low education levels (43\% had not completed high school and approximately 3 in 4 had not completed university education). Most participants were recruited from areas with socioeconomic deprivation (median IAD $5 \pm 6$, median IEO $5 \pm 6$ and median IER $4 \pm 5$ ).

AF during follow up. Over a median follow-up of 21 months (range 5-31 months), 50 patients (13\%) were diagnosed with AF. Of these 37 patients (9.8\%) were diagnosed with incident AF, 23 of whom (6\%) were diagnosed with portable ECG monitoring while 14 (4\%) were diagnosed by local physicians during the follow up period or had AF during hospitalizations. Table 2 compares the characteristics of those with AF and sinus rhythm; new onset AF was more likely in men, and in those with a higher CHARGE-AF score and those from socioeconomically deprived areas $(\mathrm{p}<0.05)$.

Association between socioeconomic status and AF risk. SEIFA data was available in 370/379 participants (with 48 AF outcomes). Table 3 summarizes the features associated with AF risk including increased age, male gender, reduced global longitudinal strain, increased left atrial volume, as well as socioeconomic deprivation. Those who developed AF had lower median SEIFA indices compared to those in sinus rhythm (IAD (4.0 (range 2-6) vs. 5.0 (range 3-8), p=0.005), IER (4.0 (range 2-5) vs. 5.0 (range 3-8), p=0.002) and IEO (3.5 (range 2-7) vs. 6.5 (range 2-8), $\mathrm{p}=0.02$ )). There were no differences in AF rates noted in participants with high school or tertiary level education. In a multivariable model, adjusted for gender, clinical risk factors, LV function and left atrial volume, increased incident AF risk was associated with disadvantaged areas (HR for IAD $=1.16$, 95\% C.I 1.05-1.29, $\mathrm{p}=0.005$ ) and areas with lower education/occupation levels (HR for IEO $=1.18$, 95\% C.I 
1.07-1.30, p=0.001) (Figure 1,3 and Table 3). Areas with lower economic resources were not independently associated with increased incident AF risk (HR for IER $=1.11$, 95\% C.I 0.99-1.24, $\mathrm{p}=0.08)$ (Figure 2).

\section{Discussion}

The results of our study suggest that a significant number of elderly people in the community with risk factors have subclinical AF. Regional socioeconomic deprivation is associated with AF independent of other clinical risk factors and cardiac function. Areas with higher household income, higher rates of education and employment were associated with reduced risk of incident AF.

Socioeconomic status and AF. Socioeconomic deprivation is well established as a risk factor for metabolic syndrome and cardiovascular disease, ${ }^{18-20}$ but the association between socioeconomic status and AF is less clear. A large Swedish study did not find an independent association between socioeconomic status and hospitalised $A F,{ }^{24}$ although an association was found in women. ${ }^{24}$ The ARIC cohort found that low family income was associated with increased risk of $\mathrm{AF},{ }^{23}$ with lower education levels associated with increased AF risk in women. ${ }^{23}$ After adjusting for confounders, we found that regional indices of low income/education were associated with AF in both sexes. The use of regional indices of socioeconomic status provide a basis of using geographic location in planning screening, in a way that using individual income or educational data would be inaccessible.

There may be several mechanisms by which socioeconomic status can affect AF risk and management. AF has been strongly associated with metabolic syndrome and obesity, ${ }^{5,7}$ both of which are strongly influenced by socioeconomic status. Household income and education levels influence dietary habits and physical activity levels. Interestingly, in our study despite high rates of obesity, T2DM and hypertension at baseline, we did not see any significant difference in these markers in participants with AF compared with those in sinus rhythm. It is possible that could be due to our small sample size and limited follow up. These markers are components of the CHARGE-AF score, where 
we did note a higher score in those with AF compared with those in sinus rhythm. It is possible that even though the overall rates between both groups were similar, those who developed AF may have poorly controlled risk factors or may have individuals with multiple risk factors present, hence creating incremental risk. Children born to parents of low socioeconomic status have higher risk of low birth weight which has been shown to be associated with AF risk. ${ }^{26}$ The higher rates of alcohol and substance abuse in areas of socioeconomic deprivation are both associated with AF risk. ${ }^{22}$ There may be added challenges such as poor health awareness in this cohort and issues relating to poor adherence, as limited household income may influence decisions made on anticoagulation and other pharmacological therapy. ${ }^{27}$ Irrespective of the mechanisms involved, socioeconomic status appears to be associated with AF and in our study, it was as important a risk factor as left atrial volume and left ventricular function.

Early diagnosis of AF and community screening. AF creates a significant burden on both patients and the health care system. For patients, it is associated with increased risk of stroke and heart failure as well as causing symptoms and impaired quality of life. AF seems likely to continue to increase in incidence and the costs to the health care system will continue to increase. ${ }^{1,3,6}$ Early diagnosis might be achieved by community screening programs, but successful AF screening requires both an appropriate diagnostic tool as well as careful selection of the at-risk population.

The incident AF rate of $9.8 \%$ in this study - higher than previously reported in the literature ${ }^{9,10,15}$ - is attributable to not only age and clinical risk factors for AF, but also social vulnerability. Elderly, socially isolated patients with poor access to affordable health care, limited household income and poor education levels are often encountered in hospital following the complications of AF. Early diagnosis offers the possibility for early initiation of treatment which may offset some of the complications which may lead to reduced hospitalizations and associated health care costs. Patients with subclinical AF and atrial tachyarrhythmias have an increased risk of stroke and cardiovascular 
events similar to those with established $\mathrm{AF}^{28,29}$ and anticoagulation may help reduce the incidence of stroke in this cohort. Active lifestyle intervention may lead to a reduction in symptom burden and in the need for repeat ablation procedures and an improvement in quality of life. ${ }^{8}$ Initiation of lifestyle intervention and risk factor modification following early diagnosis of AF may be associated with positive LA remodelling, may reduce disease progression, and may produce additional health benefits including reduction in cardiovascular risk and improvement in exercise capacity.

Clinical Implications: The results of our study have several important clinical implications. We have demonstrated a significant burden of subclinical AF in elderly people with risk factors. We have also demonstrated that AF screening using portable ECG monitoring is feasible. The finding that low regional socioeconomic status is associated with increased AF risk has important implications for mass screening. Screening programs conducted in these areas will likely yield higher detection rates, improving cost-effectiveness and providing access to early intervention programs to those at the highest risk of complications and hospitalisations.

Limitations: There are several limitations of our study. There is a potential for population selection bias as participants were recruited with newspaper and radio advertising. Selection bias may influence the rates of clinically diagnosed AF in the cohort. Patients from areas of high socioeconomic status who may have better access to health care and improved health literacy may present more frequently for review, potentially resulting in higher rates of clinically diagnosed AF. Our patient sample was small, and we had a limited number of AF outcomes. Our patient population was predominantly white Australian, and our results are not generalizable to the indigenous population or other ethnicities. Our screening for subclinical AF was done for a one-week period of intermittent ECG monitoring, and it is possible that resulted in some AF outcomes to be potentially missed. Our study focused on the assessment of regional socioeconomic status as we investigated the implications to a community AF screening program. Hence, the results of our study cannot be used for individual risk assessment. Our 
cohort study is unable to establish causality. Intervention studies are required in the future to determine if socioeconomic deprivation is a risk factor for AF and if community-based interventions can result in reduced AF incidence.

Conclusion: Elderly patients with risk factors have a high prevalence of subclinical AF, especially in regions of socioeconomic deprivation. The finding that socioeconomic deprivation is independently associated with incident AF suggests that additional resources and access to health care is needed in selected communities to improve health outcomes.

Funding: S.R is supported by a research scholarship by the Heart Foundation and Avant. This study was partially supported by Diabetes Australia, the Tasmanian Community Fund and Siemens Healthcare Australia. ECG equipment and software support were provided by Semacare Inc, a manufacturer of handheld ECG devices. The sponsors had no role in the design and conduct of the study, in the collection, analysis, and interpretation of the data, and in the preparation, review, or approval of the manuscript.

\section{Abbreviations}

AF - Atrial Fibrillation

BMI - Body Mass Index

BP - Blood Pressure

ECG - Electrocardiogram

HR - Hazard Ratio

IAD - Index of Advantage/Disadvantage 
IEO - Index of Education/Occupation

IER - Index of Economic Resources

LV - Left Ventricular

SEIFA - Socio-Economic Indices for Areas

T2DM - Type II Diabetes Mellitus

\section{References}

1 Krahn AD, Manfreda J, Tate RB, Mathewson FA, Cuddy TE. The natural history of atrial fibrillation: incidence, risk factors, and prognosis in the Manitoba Follow-Up Study. The American Journal of Medicine. 1995;98(5):476-84.

2 Vidaillet H, Granada JF, Chyou P-H, Maassen K, Ortiz M, Pulido JN, et al. A population-based study of mortality among patients with atrial fibrillation or flutter. The American Journal of Medicine. 2002;113(5):365-70.

This article is protected by copyright. All rights reserved. 
3 Kim MH, Johnston SS, Chu B-C, Dalal MR, Schulman KL. Estimation of total incremental health care costs in patients with atrial fibrillation in the United States. Circulation: Cardiovascular Quality and Outcomes. 2011;4(3):313-20.

$4 \quad$ Leyden JM, Kleinig TJ, Newbury J, Castle S, Cranefield J, Anderson CS, et al. Adelaide stroke incidence study: declining stroke rates but many preventable cardioembolic strokes. Stroke. 2013;44(5):1226-31.

5 Watanabe H, Tanabe N, Watanabe T, Darbar D, Roden DM, Sasaki S, et al. Metabolic syndrome and risk of development of atrial fibrillation. Circulation. 2008;117(10):1255-60.

6 Ball J, Thompson DR, Ski CF, Carrington MJ, Gerber T, Stewart S. Estimating the current and future prevalence of atrial fibrillation in the Australian adult population. Med J Aust. 2015;202(1):325.

7 Dagres N, Anastasiou-Nana M. Atrial Fibrillation and Obesity. Journal of the American College of Cardiology. 2010;55(21):2328-9.

8 Pathak RK, Middeldorp ME, Lau DH, Mehta AB, Mahajan R, Twomey D, et al. Aggressive risk factor reduction study for atrial fibrillation and implications for the outcome of ablation: the ARREST-AF cohort study. Journal of the American College of Cardiology. 2014;64(21):2222-31.

9 Lowres N, Neubeck L, Salkeld G, Krass I, McLachlan AJ, Redfern J, et al. Feasibility and costeffectiveness of stroke prevention through community screening for atrial fibrillation using iPhone ECG in pharmacies. The SEARCH-AF study. Thrombosis \& Haemostasis. 2014;111(6):1167-76.

10 Svennberg E, Engdahl J, Al-Khalili F, Friberg L, Frykman V, Rosenqvist M. Mass Screening for Untreated Atrial Fibrillation: The STROKESTOP Study. Circulation. 2015;131(25):2176-84.

11 Engdahl J, Andersson L, Mirskaya M, Rosenqvist M. Stepwise Screening of Atrial Fibrillation in a 75-Year-Old PopulationClinical Perspective. Circulation. 2013;127(8):930-7.

12 Kaasenbrood F, Hollander M, Rutten FH, Gerhards L, Hoes AW, Tieleman RG. Yield of screening for atrial fibrillation in primary care with a hand-held, single-lead electrocardiogram device during influenza vaccination. Europace. 2016;18(10):1514-20.

13 Proietti M, Mairesse GH, Goethals P, Scavee C, Vijgen J, Blankoff I, et al. A population screening programme for atrial fibrillation: a report from the Belgian Heart Rhythm Week screening programme. Europace. 2016:euw069.

14 Halcox JP, Wareham K, Cardew A, Gilmore M, Barry JP, Phillips C, et al. Assessment of remote heart rhythm sampling using the AliveCor heart monitor to screen for atrial fibrillation: the REHEARSE-AF study. Circulation. 2017;136(19):1784-94. 
15 Chan N, Choy C. Screening for atrial fibrillation in 13122 Hong Kong citizens with smartphone electrocardiogram. Heart. 2017;103(1):24-31.

16 Huxley RR, Lopez FL, Folsom AR, Agarwal SK, Loehr LR, Soliman EZ, et al. Absolute and Attributable Risks of Atrial Fibrillation in Relation to Optimal and Borderline Risk Factors - Clinical Perspective. Circulation. 2011;123(14):1501-8.

17 Alonso A, Krijthe BP, Aspelund T, Stepas KA, Pencina MJ, Moser CB, et al. Simple risk model predicts incidence of atrial fibrillation in a racially and geographically diverse population: the CHARGE-AF consortium. Journal of the American Heart Association. 2013;2(2):e000102.

18 Kaplan GA, Keil JE. Socioeconomic factors and cardiovascular disease: a review of the literature. Circulation. 1993;88(4):1973-98.

19 Santos AC, Ebrahim S, Barros H. Gender, socio-economic status and metabolic syndrome in middle-aged and old adults. BMC Public Health. 2008;8(1):62.

20 Chichlowska KL, Rose KM, Diez-Roux AV, Golden SH, McNeill AM, Heiss G. Individual and Neighborhood Socioeconomic Status Characteristics and Prevalence of Metabolic Syndrome. The Atherosclerosis Risk in Communities (ARIC) Study. Psychosomatic Medicine. 2008;70(9):986.

21 McLaren L. Socioeconomic status and obesity. Epidemiologic reviews. 2007;29(1):29-48.

22 Fone DL, Farewell DM, White J, Lyons RA, Dunstan FD. Socioeconomic patterning of excess alcohol consumption and binge drinking: a cross-sectional study of multilevel associations with neighbourhood deprivation. BMJ Open. 2013;3(4).

23 Misialek JR, Rose KM, Everson-Rose SA, Soliman EZ, Clark CJ, Lopez FL, et al. Socioeconomic status and the incidence of atrial fibrillation in whites and blacks: the Atherosclerosis Risk in Communities (ARIC) study. Journal of the American Heart Association. 2014;3(4):e001159.

24 Zöller B, Li X, Sundquist J, Sundquist K. Neighbourhood deprivation and hospitalization for atrial fibrillation in Sweden. Europace. 2013;15(8):1119-27.

25 Australian Bureau of Statistics. Census of Population and Housing: Socio-Economic Indexes for Areas (SEIFA), Commonwealth of Australia. [document]. Australian Bureau of Statistics, Commonwealth of Australia.; 2011 [updated 2013-03-28. Available from: http://www.abs.gov.au/ausstats/abs@.nsf/mf/2033.0.55.001.

26 Lawani SO, Demerath EW, Lopez FL, Soliman EZ, Huxley RR, Rose KM, et al. Birth weight and the risk of atrial fibrillation in whites and African Americans: the Atherosclerosis Risk In Communities (ARIC) study. BMC Cardiovascular Disorders. 2014;14(1):69. 
27 Sholzberg M, Gomes T, Juurlink DN, Yao Z, Mamdani MM, Laupacis A. The influence of socioeconomic status on selection of anticoagulation for atrial fibrillation. PloS one.

2016;11(2):e0149142.

28 Martinez C, Katholing A, Freedman SB. Adverse prognosis of incidentally detected ambulatory atrial fibrillation. Thrombosis and haemostasis. 2014;112(2):276-86.

29 Healey JS, Connolly SJ, Gold MR, Israel CW, Van Gelder IC, Capucci A, et al. Subclinical Atrial Fibrillation and the Risk of Stroke. New England Journal of Medicine. 2012;366(2):120-9.

\section{Figure Legends:}

Figure 1 - Nelson-Aalen Curves showing incident AF diagnosis based on A) IAD decile rank, B) IER decile rank and C) IEO decile rank 


\begin{tabular}{|c|c|}
\hline Demographics & $\mathbf{n}=379$ \\
\hline Age - years (SD) & $70.3(4.2)$ \\
\hline Male n (\%) & $169(45)$ \\
\hline Systolic BP mmHg (SD) & $140.6(15.9)$ \\
\hline Diastolic BP mmHg (SD) & $82.5(9.9)$ \\
\hline Heart Rate /min (SD) & $68.7(10.8)$ \\
\hline BMI (kg/m²) (SD) & $29.8(5.2)$ \\
\hline Current/Previous Smoking n (\%) & $182(48)$ \\
\hline Diabetes Mellitus n (\%) & $176(46)$ \\
\hline Obesity n (\%) & $176(46)$ \\
\hline Hypercholesterolemia n (\%) & $205(54)$ \\
\hline Hypertension n (\%) & $293(77)$ \\
\hline Previous history of IHD n (\%) & $16(4)$ \\
\hline Previous history of AF n (\%) & $13(3)$ \\
\hline Previous chemotherapy n (\%) & $36(10)$ \\
\hline Median Six-minute walk test m (IQR) & $504(96)$ \\
\hline Median CHARGE-AF \% (IQR) & $6.8(6.6)$ \\
\hline Median $\mathrm{CHA}_{2} \mathrm{DS}_{2}$-VASC \% (IQR) & $3.0(2.0)$ \\
\hline Echocardiographic Parameters & Mean (SD) \\
\hline Ejection Fraction \% (SD) & $62.8(6.2)$ \\
\hline Global Longitudinal Strain \% (SD) & $-18.8(2.5)$ \\
\hline E/e' (Average of lateral and septal) (SD) & $8.7(2.5)$ \\
\hline Left atrial volume - indexed $\mathrm{ml} / \mathrm{m}^{2}(\mathrm{SD})$ & $31.5(9.1)$ \\
\hline Left Ventricular mass - indexed $\mathrm{g} / \mathrm{m}^{2}(\mathrm{SD})$ & $88.8(21.7)$ \\
\hline Social Factors & \\
\hline
\end{tabular}




\begin{tabular}{lc}
\hline Median SEIFA Index of Advantage/Disadvantage (IQR) & $5(6.0)$ \\
Median SEIFA Index of Education/Occupation (IQR) & $5(6.0)$ \\
Median SEIFA Index of Economic Resources (IQR) & $4(5.0)$ \\
Completed High School n(\%) & $214 / 375(57)$ \\
Completed University n(\%) & $88 / 376(23)$
\end{tabular}

BMI - Body Mass Index $\quad$ BP - Blood Pressure IHD - Ischaemic Heart Disease

Table 1 - Baseline Characteristics of the overall cohort

This article is protected by copyright. All rights reserved. 
Socioeconomic status and risk of incident atrial fibrillation; Page 18

BMI - Body Mass Index BP - Blood Pressure $\quad$ IHD - Ischaemic Heart Disease

Table 2 - Comparison of baseline characteristics between patients with AF and sinus rhythm 


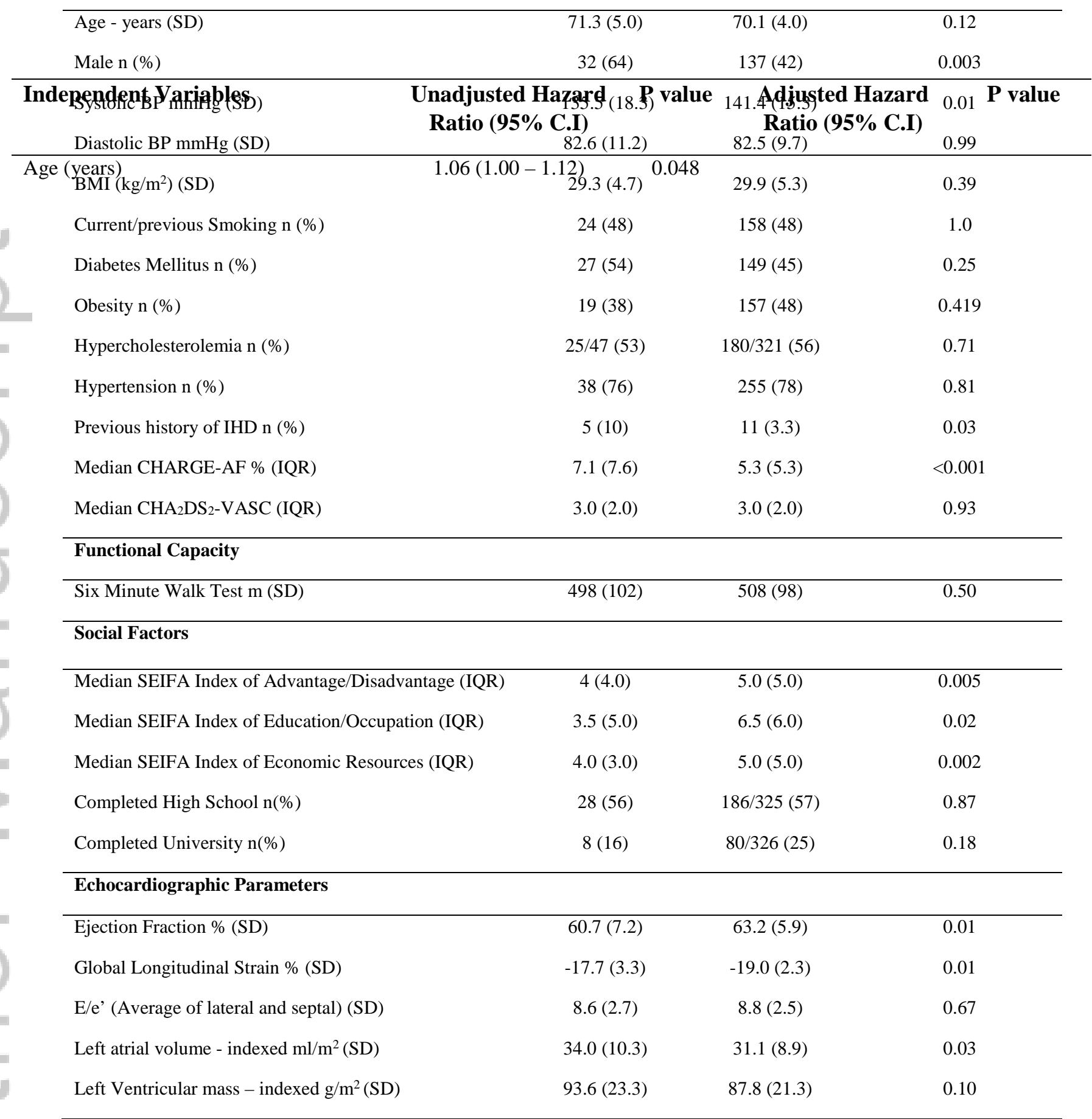




\begin{tabular}{|c|c|c|c|c|}
\hline Male Gender & $2.44(1.37-4.36)$ & 0.003 & $1.78(0.96-3.31)$ & 0.07 \\
\hline Body Mass Index $\left(\mathrm{kg} / \mathrm{m}^{2}\right)$ & $1.01(0.95-1.06)$ & 0.83 & & \\
\hline Systolic Blood Pressure (mmHg) & $0.98(0.96-0.99)$ & 0.02 & & \\
\hline Diastolic Blood Pressure (mmHg) & $1.01(0.98-1.04)$ & 0.51 & & \\
\hline Type 2 Diabetes Mellitus & $1.33(0.75-2.34)$ & 0.32 & & \\
\hline Obesity & $0.90(0.51-1.59)$ & 0.71 & & \\
\hline Hypertension & $0.99(0.52-1.91)$ & 0.99 & & \\
\hline Ejection Fraction (\%) & $0.93(0.90-0.97)$ & 0.001 & & \\
\hline Global Longitudinal Strain (\%) & $1.21(1.09-1.36)$ & 0.001 & $1.22(1.08-1.38)$ & 0.002 \\
\hline Left atrial volume - indexed $\left(\mathrm{ml} / \mathrm{m}^{2}\right)$ & $1.05(1.02-1.08)$ & 0.002 & $1.04(1.01-1.07)$ & 0.01 \\
\hline Left Ventricular mass - indexed $\left(\mathrm{g} / \mathrm{m}^{2}\right)$ & $1.01(0.99-1.02)$ & 0.02 & & \\
\hline Six Minute Walk Test (m) & $1.00(0.99-1.00)$ & 0.87 & & \\
\hline CHARGE-AF Score (\%) & $1.04(0.99-1.09)$ & 0.10 & $0.98(0.92-1.03)$ & 0.37 \\
\hline $\mathrm{CHA}_{2} \mathrm{DS}_{2}-\mathrm{VASC}$ Score & $0.98(0.71-1.34)$ & 0.89 & & \\
\hline SEIFA Index of Advantage/Disadvantage & $1.15(1.04-1.27)$ & 0.007 & $1.16(1.05-1.29)$ & 0.005 \\
\hline SEIFA Index of Education/Occupation & $1.17(1.06-1.28)$ & 0.001 & $1.18(1.07-1.30)$ & 0.001 \\
\hline SEIFA Index of Economic Resources & $1.11(0.99-1.25)$ & 0.07 & $1.11(0.99-1.24)$ & 0.08 \\
\hline Completed High School & $0.67(0.39-1.18)$ & 0.17 & & \\
\hline Completed University & $0.55(0.26-1.16)$ & 0.12 & & \\
\hline
\end{tabular}

Table 3 - Cox regression analysis showing association between socioeconomic status and AF. Multivariable model adjusted for gender, CHARGE-AF score, Left atrial volume and Global Longitudinal strain. 

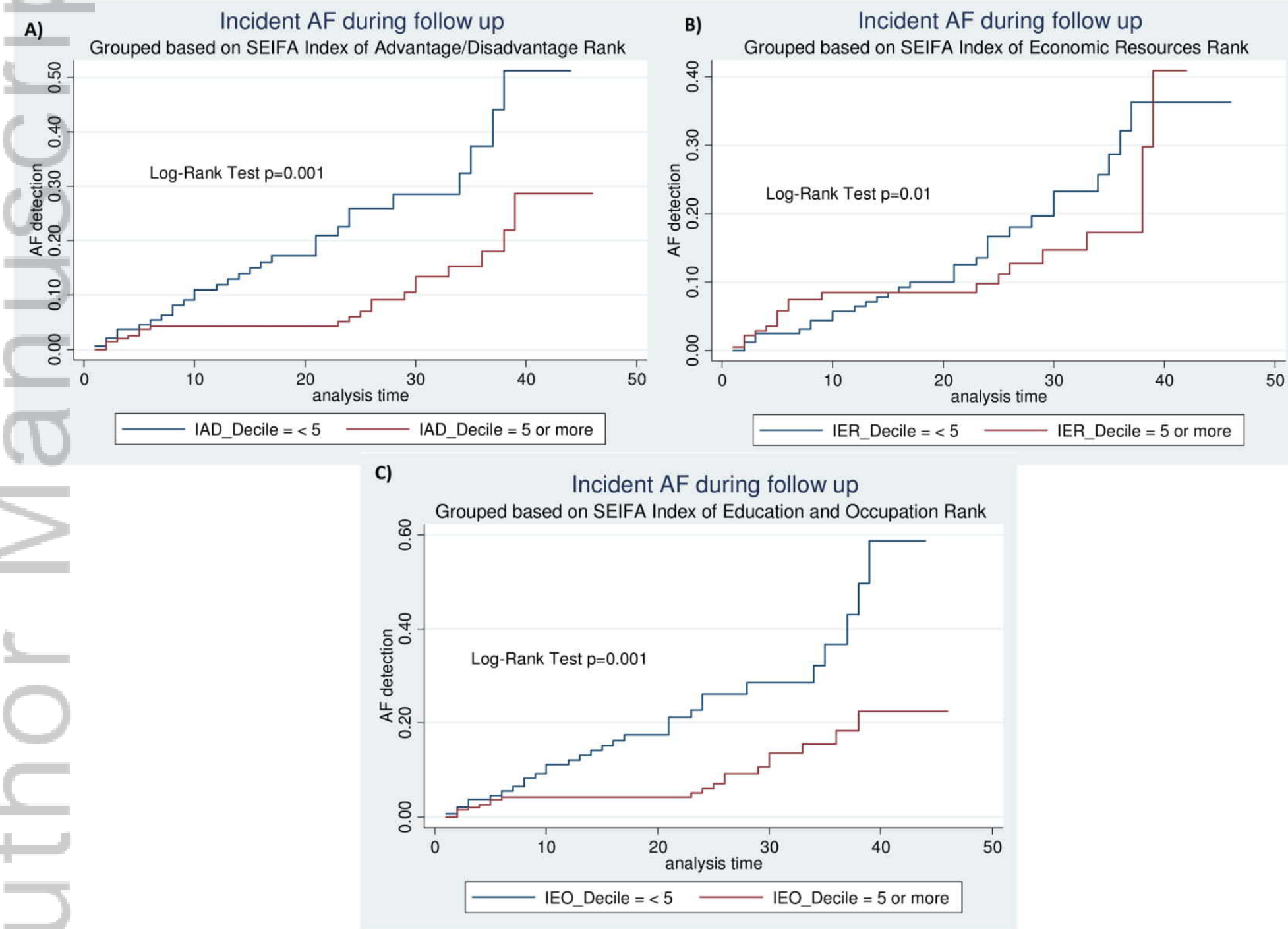

IMJ_14214_Marwick---IMJ-0887-2018-Figure-1-combined-1.jpg

This article is protected by copyright. All rights reserved. 


\section{University Library}

\section{- M M N E R VA A gateway to Melbourne's research publications}

Minerva Access is the Institutional Repository of The University of Melbourne

Author/s:

Ramkumar, S;Ochi, A;Yang, H;Nerlekar, N;D'Elia, N;Potter, EL;Murray, IC;Nattraj, N;Wang, Y;Marwick, TH

Title:

Association between socioeconomic status and incident atrial fibrillation

Date:

2019-10-01

\section{Citation:}

Ramkumar, S., Ochi, A., Yang, H., Nerlekar, N., D'Elia, N., Potter, E. L., Murray, I. C., Nattraj, N., Wang, Y. \& Marwick, T. H. (2019). Association between socioeconomic status and incident atrial fibrillation. INTERNAL MEDICINE JOURNAL, 49 (10), pp.1244-1251. https:// doi.org/10.1111/imj. 14214 .

Persistent Link:

http://hdl.handle.net/11343/286460 